\title{
EXTREMAL PROBLEMS AND COEFFICIENT REGIONS FOR ANALYTIC FUNCTIONS REPRESENTED BY A STIELTJES INTEGRAL ${ }^{(1)}$
}

\section{BY \\ J. A. PFALTZGRAFF}

1. Introduction. Let $D$ be a simply-connected region and let $\mathscr{S}=\mathscr{S}(g, D)$ denote the class of functions $f(z)$ analytic in $D$ and represented by the Stieltjes integral

$$
f(z)=\int_{0}^{2 \pi} g(z, t) d \mu(t), \quad z \in D
$$

where $\mu(t)$ is a monotone nondecreasing function on the interval $0 \leqq t \leqq 2 \pi$, normalized by the condition

$$
\int_{0}^{2 \pi} d \mu(t)=1
$$

and $g(z, t)$ is a given function with the following properties:

(1.1b) $g(z, t)$ is analytic with respect to $z$ in $D$.

(1.1c) The functions

$$
g^{(k)}(z, t) \frac{d^{(k)} g(z, t)}{d z^{k}}, \quad k=0,1, \cdots
$$

are continuous with respect to $t$ in $0 \leqq t \leqq 2 \pi$.

Let $\mathscr{P}$ denote the class of functions

$$
P(z)=1+p_{1} z+p_{2} z^{2}+\cdots, \quad P(0)=1,
$$

which are regular and have positive real part in $|z|<1$. It is well known [5] that

$$
\mathscr{P}=\mathscr{S}\left(\frac{1+z e^{-i t}}{1-z e^{-i t}},|z|<1\right) .
$$

A structural formula of the type (1.1) for the class $\mathscr{P}(\mathscr{E})$ of functions which are regular and have positive real part in an ellipse $\mathscr{E}$ has been obtained by Royster [9].

In this paper we study a rather general type of extremal problem in each of the classes $\mathscr{S}(g,|z|<1)$ and $\mathscr{P}$, and in $\$ 4$ we obtain a characteri-

Presented to the Society, April 25, 1964; received by the editors March 6, 1964.

( ${ }^{1}$ ) This work is based on a portion of the author's doctoral dissertation written under the direction of Professor W. C. Royster of the University of Kentucky, during the tenure of a Haggin Fellowship. 
zation of the coefficient regions of functions in the class $\mathscr{P}(\mathscr{E})$. With the exception of \$3, where a simple type of variational technique is used, the methods employed in this paper are elementary and depend primarily upon properties of the Stieltjes integral (1.1) and properties of convex sets in real, finite-dimensional Euclidean space.

2. Value regions of $\mathscr{S}(g,|z|<1)$. Let $\mathscr{S}=\mathscr{S}(g,|z|<1)$ and for a given integer $n \geqq 0$ let

$$
v(f, z)=\left(f(z), z f^{\prime}(z), \cdots, z^{n} f^{(n)}(z)\right),
$$

where $z(|z|<1)$ is fixed and $f \in \mathscr{S}$, then corresponding to each $f \in \mathscr{Y}$, $v(f, z)$ can be considered as a point in $(2 n+2)$-dimensional real Euclidean space $R^{2 n+2}$. Let

$$
V_{n}=\{v(f, z): f \in \mathscr{S}\} .
$$

Then $V_{n}$ is called the value region of $v(f, z)$ in the class $\mathscr{S}$. Observe that $V_{0}$ is the value region of $f(z)$ in the class $\mathscr{S}$ (compare [1]). The following three theorems are generalizations of results due to Ašnevic and Ulina [1].

Theorem 2.1. The value region $V_{n}$ of $v(f, z)$ in the class $\mathscr{S}$ is a closed connected convex set.

Proof. $V_{n}$ is closed since the class $\mathscr{S}$ is compact. Let $v_{1}, v_{2}$ be points in $V_{n}$ then $v_{k}=v\left(f_{k}, z\right)$ for some $f_{k} \in \mathscr{S}, k=1,2$. For any $\lambda, 0 \leqq \lambda \leqq 1$ the function $f_{\lambda}=f_{1}+(1-\lambda) f_{2}$ belongs to the class $\mathscr{S}$, [1, Corollary, p. 82]. If we let $v_{\lambda}=v\left(f_{\lambda}, z\right)$, then $v_{\lambda} \in V_{n}$, and by (2.1) $v_{\lambda}=\lambda v_{1}+(1-\lambda) v_{2}$. As $\lambda$ varies from zero to one the point $v_{\lambda}$ describes the line segment joining $v_{1}$ and $v_{2}$, and therefore, $V_{n}$ is both convex and connected.

Before proving the next theorem we state two known results on convex sets in $R^{m}, m$-dimensional real Euclidean space, (see [3, Chapters 1, 2]). Throughout the paper $\mathscr{H}(\mathscr{A})$ denotes the convex hull of a set $\mathscr{A}$.

(A) If $\mathscr{A}$ is a compact subset of $R^{m}$ then $\mathscr{H}(\mathscr{C})$ is compact

(B) (Carathéodory) If $\mathscr{A} \subset R^{m}$ and $\mathscr{A}$ has $m$ or less components then $\mathscr{H}(\mathscr{A})$ consists of all points expressible in the form $\sum_{1}^{r} \lambda_{k} x_{k}$ where $r \leqq m$, the $x_{k}$ are any points of $\mathscr{A}, \lambda_{k}>0$ and $\sum_{1}^{r} \lambda_{k}=1$. The index $r$ is not fixed.

Let $V_{n}(g)$ denote the set of points

$$
v(g, z)=\left(g(z, t), \cdots, z^{n} g^{(n)}(z, t)\right), \quad 0 \leqq t \leqq 2 \pi,
$$

where $g(z, t)$ is the function given in (1.1). By (1.1c), $V_{n}(g)$ is a compact connected subset of $R^{2 n+2}$. Moreover, by (A) and (B), $\mathscr{H}\left(V_{n}(g)\right)$ is compact and consists of all points of the form

$$
\sum_{k=1}^{r} \lambda_{k}\left(g\left(z, t_{k}\right), \cdots, z^{n} g^{(n)}\left(z, t_{k}\right)\right),
$$


where $r \leqq 2 n+2, \lambda_{k}>0, \sum_{1}^{r} \lambda_{k}=1$, and $0 \leqq t_{k} \leqq 2 \pi$.

TheOREM 2.2. The value region of $v(f, z)$ in $\mathscr{S}$ is the convex hull of $V_{n}(g)$, i.e., $V_{n}=\mathscr{H}\left(V_{n}(g)\right)$.

Proof. Let $x \in \mathscr{A}\left(V_{n}(g)\right)$ then $x$ is a point of the form given in (2.4). For each $k, 1 \leqq k \leqq r$, define a step function

$$
\mu_{k}(t)= \begin{cases}0, & 0 \leqq t<t_{k}, \\ 1, & t_{k} \leqq t \leqq 2 \pi,\end{cases}
$$

and let $\beta_{1}(t)=\mu_{1}(t), \beta_{2}(t)=\left(1-\lambda_{1}\right)^{-1} \sum_{2}^{r} \lambda_{k} \mu_{k}(t)$. Then $\beta_{1}$ and $\beta_{2}$ are nondecreasing functions of $t$ which satisfy (1.1a), and the functions

$$
f_{1}(\zeta)=\int_{0}^{2 \pi} g(\zeta, t) d \beta_{1}(t)=g\left(\zeta, t_{1}\right)
$$

and

$$
f_{2}(\zeta)=\int_{0}^{2 \pi} g(\zeta, t) d \beta_{2}(t)=\left(1-\lambda_{1}\right)^{-1} \sum_{2}^{r} \lambda_{k} g\left(\zeta, t_{k}\right)
$$

belong to $\mathscr{S}$. Thus $x \in V_{n}$ since $x=\lambda_{1} v\left(f_{1}, z\right)+\left(1-\lambda_{1}\right) v\left(f_{2}, z\right)$ and $V_{n}$ is convex. We have shown that $\mathscr{U}\left(V_{n}(g)\right) \subset V_{n}$.

Now assume that $x \in V_{n}$, then $x=v(f, z)$ for some $f(\zeta)=\int_{0}^{2 \pi} g(\zeta, t) d \mu(t)$ $\in \mathscr{S}$. If $\mu(t)$ is a step function with a finite number of jumps, say $m$, then

$$
f(\zeta)=\sum_{1}^{m} \lambda_{k} g\left(\zeta, t_{k}\right), \quad \lambda_{k}>0, \quad \sum_{1}^{m} \lambda_{k}=1,
$$

and $x \in \mathscr{H}\left(V_{n}(g)\right)$ since

$$
x=\sum_{1}^{m} \lambda_{k}\left(g\left(z, t_{k}\right), \cdots, z^{n} g^{(n)}\left(z, t_{k}\right)\right)
$$

is a convex combination of points $\left(g\left(z, t_{k}\right), \cdots, z^{n} g^{(n)}\left(z, t_{k}\right)\right)$ from $V_{n}(g)$. If this is not the case then we can show that $x$ is a limit point of $\mathscr{M}\left(V_{n}(g)\right)$ and consequently is also a member of $\mathscr{Z}\left(V_{n}(g)\right)$ since $\mathscr{K}\left(V_{n}(g)\right)$ is a compact subset of $R^{2 n+2}$.

Let $\epsilon>0$ be given. Then corresponding to each $k=0,1, \cdots, n$, there is a partition

$$
P(k): 0=t_{0}^{(k)}<t_{1}^{(k)}<\cdots<t_{m(k)}^{(k)}=2 \pi
$$

of $0 \leqq t \leqq 2 \pi$ such that

$$
\left|f^{(k)}(z)-\sum_{j=1}^{M(k)} g^{(k)}\left(z, t_{j}^{(k)}\right) \Delta_{j}^{(k)} \mu\right|^{2}<\frac{\epsilon^{2}}{n+1},
$$


where $\Delta_{j}^{(k)} \mu=\mu\left(t_{j}^{(k)}\right)-\mu\left(t_{j-1}^{(k)}\right), j=1, \cdots, M(k)$. This follows from the fact that $f^{(k)}(z)=\int_{0}^{2 \pi} g^{(k)}(z, t) d \mu(t)$ and the definition of the Stieltjes integral. Recall that $z,|z|<1$, is fixed and let

$$
P: 0=t_{0}<t_{1}<\cdots<t_{m}=2 \pi
$$

denote the partition obtained from the union of the $n+1$ partitions $P(k)$, then the inequality

$$
\left|f^{(k)}(z)-\sum_{j=1}^{m} \lambda_{j} g^{(k)}\left(z, t_{j}\right)\right|^{2}<\frac{\epsilon^{2}}{n+1}
$$

$\left(\lambda_{j}=\mu\left(t_{j}\right)-\mu\left(t_{j-1}\right)\right)$ holds for each $k=0,1, \cdots, n$. By (1.1a) $\sum_{1}^{m} \lambda_{j}=1$ and hence

$$
y=\sum_{j=1}^{m} \lambda_{j}\left(g\left(z, t_{j}\right), \cdots, z^{n} g^{(n)}\left(z, t_{j}\right)\right) \in \mathscr{H}\left(V_{n}(g)\right)
$$

since it is a convex combination of points belonging to $V_{n}(g)$. If we let $d$ denote the metric in $R^{2 n+2}$ then by (2.5)

$$
\begin{aligned}
d(x, y) & =\left\{\left|f(z)-\sum_{j=1}^{m} \lambda_{j} g\left(z, t_{j}\right)\right|^{2}+\cdots+\left|z^{n} f^{(n)}(z)-\sum_{j=1}^{m} \lambda_{j} z^{n} g^{(n)}\left(z, t_{j}\right)\right|^{2}\right\}^{1 / 2} \\
& <\epsilon,
\end{aligned}
$$

where we have used the fact that $|z|<1$. Thus $x$ is a limit point of $\mathscr{H}\left(V_{n}(g)\right)$. This completes the proof of Theorem 2.2.

It is easy to see that the preceding results also hold for the more general type of value region which consists of points of the form

$$
\left(c_{0} f(z), c_{1} z^{m(1)} f^{\prime}(z), \cdots, c_{n} z^{m(n)} f^{(n)}(z)\right),
$$

where $c_{k}(k=0,1, \cdots, n)$ are givén constants and $m(1), m(2), \cdots, m(n)$ are given integers. In particular, if $c_{k}=(k !)^{-1}, k=0,1, \cdots, n, m(1)$ $=m(2)=\cdots=m(n)=0$, and $z=0$ then the value region is the coefficient region

$$
\mathscr{X}_{n}=\left\{\left(a_{0}, a_{1}, \cdots, a_{n}\right): f(\zeta)=\sum_{k=0}^{\infty} a_{k} \zeta^{k}\right\}
$$

of the class $\mathscr{S}(g,|z|<1)$. If $b_{k}(t), k=0,1, \cdots$, are the coefficients in the Maclaurin series of $g(z, t)$ then the coefficient region $\mathscr{X}_{n}$ is the convex hull of the set $\left\{\left(b_{0}(t), b_{1}(t), \cdots, b_{n}(t)\right): 0 \leqq t \leqq 2 \pi\right\}$.

Theorem 2.2 may be used to obtain a characterization of the extremal functions for a rather general type of extremal problem in $\mathscr{S}$. 
THeOREM 2.3. Let $F\left(u_{0}, u_{1}, \cdots, u_{n}\right)$ be a continuous function of $n+1$ complex variables in some region containing the value region $V_{n}$ of $v(f, z)$ in $\mathscr{S}$. For a given $z,|z|<1$, the minimum (maximum) of the functional

$$
J[f(z)]=\operatorname{Re}\left\{F\left(f(z), z f^{\prime}(z), \cdots, z^{n} f^{(n)}(z)\right)\right\}
$$

in the class $\mathscr{S}$ is attained for a function of the form

$$
f_{0}(\zeta)=\sum_{k=1}^{2 n+2} \lambda_{k} g\left(\zeta, t_{k}\right),
$$

where $\lambda_{k} \geqq 0$ and $\sum_{k=1}^{2 n+2} \lambda_{k}=1$.

Proof. The problem is equivalent to that of finding the minimum of the continuous real-valued function $J[f(z)]$ on the closed set $V_{n}$. Let $q_{0}$ be the point in $V_{n}$ at which the minimum is attained then since $V_{n}=\mathscr{K}\left(V_{n}(g)\right)$ we have by (2.4) that

$$
q_{0}=\sum_{k=1}^{2 n+2} \lambda_{k}\left(g\left(z, t_{k}\right), \cdots, z^{n} g^{(n)}\left(z, t_{k}\right)\right),
$$

where $\lambda_{k} \geqq 0$ and $\sum_{1}^{2 n+2} \lambda_{k}=1$. It follows that the minimum is attained for a function of the form given in (2.7).

It is clear that the complete solution to the extremal problem $J[f(z)]$ $=\min (\max )$ is obtained only when the precise values of the $4 n+4$ parameters $\lambda_{k}, t_{k}$ are determined. In the next section we require that $F\left(u_{0}, \cdots, u_{n}\right)$ be analytic and then show that the number of parameters in the solution to the extremal problem in the class $\mathscr{P}$ reduces to $2 n+2$.

3. An extremal problem for the class $\mathscr{P}$. Let $\mathscr{P}$ denote the normalized class of analytic functions

$$
P(z)=1+p_{1} z+p_{2} z^{2}+\cdots, \quad P(0)=1,
$$

which are regular and have $\operatorname{Re} P(z)>0$ in $|z|<1$. If $P(z) \in \mathscr{P}$ then $P(z)$ may be represented by the Herglotz-Stieltjes integral formula [5]

$$
P(z)=\int_{0}^{2 \pi} h(z, t) d \mu(t),
$$

where

$$
h(z, t)=\left(1+z e^{-i t}\right)\left(1-z e^{-i t}\right)^{-1}
$$

and $\mu(t)$ is a nondecreasing function in $[0,2 \pi]$, normalized so that

$$
\int_{0}^{2 \pi} d \mu(t)=1 .
$$


In other words $\mathscr{P}=\mathscr{Y}(h(z, t),|z|<1)$. We shall assume that $\mu(t)$ is continued by the formula $\mu(t+2 \pi)=\mu(t)+1$ then, since $h(z, t)$ is periodic in $t$ with period $2 \pi$, the structural formula $P(z)=\int_{a}^{b} h(z, t) d \mu(t)$ defines functions in the class $\mathscr{P}$ as long as $b-a=2 \pi$.

In Theorem 3.1 we generalize from 2 to $n+1$ variables a theorem of Robertson [7, Theorem 1]. It was known to the author that this result had previously been obtained by W. E. Kirwan who extended the method of interior variations employed by Robertson [7]. The present proof depends upon the results of Theorem 2.3 and a rather simple type of variational technique. An alternative proof of Theorem 3.1 can be obtained from a direct application of the variational techniques of Goluzin [4].

THEOREM 3.1. Let $F\left(u_{0}, u_{1}, \cdots, u_{n}\right)$ be analytic in some region that contains the value region $V_{n}$ of $v(P, z)=\left(P(z), z P^{\prime}(z), \cdots, z^{n} P^{(n)}(z)\right)$ in the class $\mathscr{P}$. For given $r, 0<r<1$, the $\min (\max )$ on $|z|=r$ of the functional

$$
J[P(z)]=\operatorname{Re}\left\{F\left(P(z), z P^{\prime}(z), \cdots, z^{n} P^{(n)}(z)\right)\right\}
$$

in the class $\mathscr{P}$ is attained for a function of the form

$$
P_{0}(z)=\sum_{\nu=1}^{n+1} \lambda_{\nu} \frac{1+z e^{-i t_{\nu}}}{1-z e^{-i t_{\nu}}},
$$

where $\lambda_{v} \geqq 0$ and $\sum_{v=1}^{n+1} \lambda_{\nu}=1$. We exclude from consideration the case in which for the extremal function the partial derivatives $F_{k}=\left(\partial F / \partial u_{k}\right)(k=0,1, \cdots, n)$ all vanish.

The author has been informed that the last restriction on the extremal problem can be removed by means of a simple type of variation in the class $\mathscr{P}$ (oral communication from Kirwan).

Proof. By Theorem 2.3 the extreme values of the functional $J[P(z)]$ are attained for functions of the form

$$
P_{0}(z)=\sum_{\nu=1}^{m} \lambda_{\nu} h\left(z, t_{\nu}\right), \quad m \leqq 2 n+2,
$$

where $\lambda_{v}>0$ and $\sum_{v=1}^{m} \lambda_{v}=1$. Here, and throughout the proof we may assume that $0 \leqq t_{1}<t_{2}<\cdots<t_{m}<2 \pi$. Note that the convex structure of the value region and the continuity of $F\left(u_{0}, u_{1}, \cdots, u_{n}\right)$ determine that the degree of $P_{0}(z)$ in (3.7) shall be no greater than $2 n+2$ (compare remarks $[7$, p. 242]).

In order to complete the proof we shall apply two types of variations to the functions (3.7) (compare [4]). Let

$$
P_{0}(z)=\sum_{\nu=1}^{m} \lambda_{\nu} h\left(z, t_{\nu}\right)
$$


and for a given integer $\eta, 1 \leqq \eta \leqq m$, define

$$
t_{v}^{*}= \begin{cases}t_{\nu}+\epsilon, & \nu=\eta, \\ t_{\nu}, & \nu \neq \eta,\end{cases}
$$

then for sufficiently small real $\epsilon$ the function

$$
P_{0}^{*}(z)=\sum_{\nu=1}^{m} \lambda_{\nu} h\left(z, t_{\nu}^{*}\right)
$$

is of the form (3.7). Using the Taylor series for $h(z, t)$,

$$
h(z, t+\epsilon)=h(z, t)+\epsilon h_{t}(z, t)+O\left(\epsilon^{2}\right),
$$

we have

$$
P_{0}^{*}(z)=P_{0}(z)+\lambda_{\eta} \epsilon h_{t}\left(z, t_{\eta}\right)+O\left(\epsilon^{2}\right) .
$$

This is the first of our variational formulas for functions of the form (3.7). Using the notation

$$
g_{t}^{(k)}(z, t)=\frac{d^{k}}{d z^{k}}\left(g_{t}(z, t)\right),
$$

and differentiating (3.8) we obtain the variational formulas

$$
P_{0}^{*(k)}(z)=P_{0}^{(k)}(z)+\epsilon \lambda_{\eta} h_{t}^{(k)}\left(z, t_{\eta}\right)+O\left(\epsilon^{2}\right),
$$

$k=0,1, \cdots, n, 1 \leqq \eta \leqq m$. The $O\left(\epsilon^{2}\right)$ term is uniformly small on compact subsets of $|z|<1$.

The second type of variational formula for the functions (3.7) is obtained by varying two of the $\lambda_{\nu}$. For a given integer $\eta, 1<\eta \leqq m+1$, $\left(\lambda_{m+1}=\lambda_{1}\right.$, and $\left.t_{m+1}=t_{1}+2 \pi\right)$ let $\lambda_{\eta-1}^{* *}=\lambda_{\eta-1}+\epsilon, \lambda_{\eta}^{* *}=\lambda_{\eta}-\epsilon$, and $\lambda_{\eta}^{* *}=\lambda_{\eta}$, $\nu \neq \eta-1, \eta$, then for sufficiently small real $\epsilon, 0<\lambda_{\nu}^{* *} \leqq 1, \lambda_{1}^{* *}+\cdots+\lambda_{m}^{* *}$ $=1$ and the function

$$
P_{0}^{* *}(z)=\sum_{\nu=1}^{m} \lambda_{\nu}^{* *} h\left(z, t_{\nu}\right)=P_{0}(z)+\epsilon\left[h\left(z, t_{\eta-1}\right)-h\left(z, t_{\eta}\right)\right]
$$

is of the form (3.7) if $P_{0}(z)$ is of that form. Thus we have the second set of variational formulas

$$
P_{0}^{* *(k)}(z)=P_{0}^{(k)}(z)+\epsilon\left[h^{(k)}\left(z, t_{\eta-1}\right)-h^{(k)}\left(z, t_{\eta}\right)\right]
$$

for the functions (3.7).

We now apply the variational formulas (3.9) and (3.11) to.show that if $J\left[P_{0}(z)\right]$ is a min $(\max )$ for a function $P_{0}(z)$ of the form (3.7) then $m \leqq n+1$. Since the class is invariant with respect to a rotation of the $z$-plane, and we are seeking the minimum on $|z|=r$ of $J[P(z)]$ it is sufficient to let $z=r>0[7$, p. 240]. 
The Taylor series for $F\left(u_{0}, \cdots, u_{n}\right)$ gives

$$
F\left(a_{0}+\alpha_{0}, \cdots, a_{n}+\alpha_{n}\right)=F\left(a_{0}, \cdots, a_{n}\right)+\alpha_{0} F_{0}+\cdots+\alpha_{n} F_{n}+\cdots,
$$

where $F_{k}=\left.\left(\partial F / \partial u_{k}\right)\right|_{\left(a_{0}, \cdots, a_{n}\right)}, k=0,1, \cdots, n$. Let

$$
a_{k}=r^{k} P_{0}^{(k)}(r), \quad \alpha_{k}=\epsilon \lambda_{\eta} r^{k} h_{t}^{(k)}\left(r, t_{\eta}\right)+O\left(\epsilon^{2}\right) .
$$

Then by (3.9),

$$
\begin{aligned}
& F\left(P_{0}^{*}(r), \cdots, r^{n} P_{0}^{*(n)}(r)\right)=F\left(P_{0}(r), \cdots, r^{n} P_{0}^{(n)}(r)\right) \\
&+\epsilon \lambda_{\eta}\left[h_{t}\left(r, t_{\eta}\right) F_{0}+\cdots+r^{n} h_{t}^{(n)}\left(r, t_{\eta}\right) F_{n}\right]+O\left(\epsilon^{2}\right),
\end{aligned}
$$

and, taking the real part,

$$
\delta J\left[P_{0}\right]=\left.\epsilon \lambda_{\eta} \Psi^{\prime}(t)\right|_{t=t_{\eta}}+O\left(\epsilon^{2}\right),
$$

where $\Psi^{\prime}(t)$ is the derivative with respect to $t$ of the function

$$
\Psi(t)=\operatorname{Re}\left\{h(r, t) F_{0}+\cdots+r^{n} h^{(n)}(r, t) F_{n}\right\},
$$

and $\delta J\left[P_{0}\right]=J\left[P_{0}^{*}(r)\right]-J\left[P_{0}(r)\right]$. If $J[P(r)]$ is a minimum for $P(z)$ $=P_{0}(z)$ then $\delta J\left[P_{0}\right] \geqq 0$ and by (3.14)

$$
\Psi^{\prime}\left(t_{\eta}\right)=0, \quad \eta=1,2, \cdots, m,
$$

since $\epsilon$ may be either positive or negative and $\lambda_{\eta}>0$.

Following the same procedure with the variational formulas (3.11) we have that

$$
\delta J\left[P_{0}\right]=\epsilon\left[\Psi\left(t_{\eta-1}\right)-\Psi\left(t_{\eta}\right)\right]+O\left(\epsilon^{2}\right), \quad 1<\eta \leqq m+1,
$$

and, since $\epsilon$ may be either positive or negative, the extremal function $P_{0}$ must satisfy the conditions

$$
\Psi\left(t_{\eta-1}\right)=\Psi\left(t_{\eta}\right), \quad 1<\eta \leqq m+1 .
$$

It follows from (3.18) that $\Psi^{\prime}(t)$ must vanish at least once in each of the intervals $\left(t_{1}, t_{2}\right),\left(t_{2}, t_{3}\right), \cdots,\left(t_{m}, t_{1}+2 \pi\right)$, and this fact with (3.16) implies that $\Psi^{\prime}(t)$ must have at least $2 m$ zeros in $0 \leqq t<2 \pi$. It remains to show that $\Psi^{\prime}(t)$ can have at most $2 n+2$ zeros in $0 \leqq t<2 \pi$, and, consequently, that $P_{0}(z)$ in (3.7) is an extremal function only if $m \leqq n+1$. Using (3.3) and (3.15) it is easy to show that the equation $\Psi^{\prime}(t)=0$ is equivalent to the equation $Q(t)=0$, where

$$
Q(t)=\operatorname{Im}\left\{\left[\sum_{k=0}^{n}(-1)^{n-k} k ! F_{k} r^{n+1-k}\right] e^{-i(n+1) t}+\cdots+(-1)^{n+2} F_{1} r^{n+2} e^{i(n+1) t}\right\}
$$

is a polynomial of degree $\leqq 2(n+1)$ in $e^{i t}$. Hence $\Psi^{\prime}(t)$ has at most $2 n+2$ zeros. 
4. Coeficient regions of functions with positive real part in an ellipse. Let $\mathscr{E}$ be an ellipse with foci at \pm 1 and semi-axes $a>b, a>1$. Points on the boundary of $\mathscr{E}$ can be represented parametrically by $z=\cos (t-i c), 0 \leqq t$ $\leqq 2 \pi$, where $a=\cosh c, b=\sinh c, c>0$. If $f(z)$ is analytic in $\mathscr{E}$ then $f(z)$ can be represented by a series of Tchebychef polynomials

$$
f(z)=\sum_{k=0}^{\infty} a_{k} T_{k}(z), \quad T_{k}(z)=\cos (k \arccos z), \quad z \in \mathscr{E},
$$

which converges uniformly interior to $\mathscr{E}[10]$. Let $\mathscr{P}(\mathscr{E})$ denote the class of functions (4.1) that are regular, have positive real part, $z \in \mathscr{E}$, and are normalized by the condition $a_{0}=1$.

Royster [8] proved that if $f(z) \in \mathscr{P}(\mathscr{\delta})$ then the $n$th coefficient $a_{n}=\alpha_{n}$ $+i \beta_{n}$ in the expansion (4.1) satisfies the sharp inequality

$$
\alpha_{n}^{2} \cosh ^{2} n c+\beta_{n}^{2} \sinh ^{2} n c \leqq 4, \quad n=1,2, \cdots .
$$

Equality in (4.2) holds for the functions

$$
\begin{aligned}
K(z, t) & =1+\sum_{k=1}^{\infty} b_{k}(t) T_{k}(z), \quad z \in \mathscr{E}, \\
b_{k}(t) & =2 \frac{\cos k t}{\cosh k c}-i 2 \frac{\sin k t}{\sinh k c}, \quad 0 \leqq t<2 \pi,
\end{aligned}
$$

which belong to $\mathscr{P}(\mathscr{E})$ and map $\mathscr{E}$ onto the right half-plane [9]. In this section we obtain characterizations of the coefficient region

$$
\mathscr{A}_{2 n}=\left\{\left(a_{1}, a_{2}, \cdots, a_{n}\right): f(z)=1+\sum a_{k} T_{k}(z) \in \mathscr{P}(\mathscr{E})\right\}
$$

and the subset $\mathscr{A}_{n-1}$ of $\mathscr{A}_{2 n}$ which consists of points $\left(a_{1}, \cdots, a_{n}\right)$ corresponding to the extremal functions $f(z) \in \mathscr{P}(\mathscr{E})$ for which $\operatorname{Re}\left(a_{n}\right)$ is a maximum. Our results parallel those of the Carathéodory theory for the class $\mathscr{P},[2],[6]$, and are derived from a Stieltjes integral representation formula for $\mathscr{P}(\mathscr{E})$.

Theorem 4.1. $\mathscr{A}_{2 n}$ is a closed connected convex subset of 2 -dimensional real Euclidean space.

Proof. $\mathscr{A}_{2 n}$ is closed since the class $\mathscr{P}(\mathscr{E})$ is compact. $\mathscr{A}_{2 n}$ is convex since $\mathscr{P}(\mathscr{E})$ is a convex class of functions. The connectedness follows immediately.

In order to obtain a more complete characterization of $\mathscr{A}_{2 n}$ we need the following result proved by Royster [9]:

If $f(z) \in \mathscr{P}(\mathscr{8})$ then $f(z)$ may be represented by the Stieltjes integral formula

$$
f(z)=\int_{0}^{2 \pi} K(z, t) d \mu(t), \quad z \in \mathscr{E}
$$


where $\mu(t)$ is a nondecreasing function with total weight one on $0 \leqq t \leqq 2 \pi$ and $K(z, t)$ is defined in (4.3), (4.4). Conversely, any function of the form (4.6) belongs to $\mathscr{P}(\mathscr{E})$.

TheOREM 4.2. $\mathscr{A}_{2 n}$ is the convex hull of the set

$$
\mathscr{D}_{2 n}=\left\{\left(b_{1}(t), \cdots, b_{n}(t)\right): 0 \leqq t \leqq 2 \pi\right\},
$$

where the $b_{k}(t)(k=1, \cdots, n)$ are defined in (4.4).

We omit the details of the proof which are essentially the same as those in the proof of Theorem 2.2. Note that if $f(z)$ in (4.1) belongs to $\mathscr{P}(\mathscr{E})$ then by (4.6)

$$
a_{k}=\int_{0}^{2 \pi} b_{k}(t) d \mu(t), \quad k=1,2, \cdots,
$$

and consequently, the points $\left(f(z), z f^{\prime}(z), \cdots, z^{n} f^{(n)}(z)\right)$ and

$$
\left(g(z, t), z g^{\prime}(t), \cdots, z^{n} g^{(n)}(z, t)\right)
$$

in Theorem 2.2 are replaced, respectively, by $\left(a_{1}, a_{2}, \ldots, a_{n}\right)$ and $\left(b_{1}(t), b_{2}(t), \cdots, b_{n}(t)\right)$ in the present theorem.

By Theorem 4.2, points in $\mathscr{A}_{2 n}$ are of the form

$$
\sum_{j=1}^{r} \lambda_{j}\left(b_{1}\left(t_{j}\right), \cdots, b_{n}\left(t_{j}\right)\right),
$$

where $\lambda_{j}>0, \sum_{j=1}^{r} \lambda_{j}=1$, and $r \leqq 2 n$. Corresponding to each point of the form (4.9) there is at least one function $f(z)=1+\sum a_{k} T_{k}(z) \in \mathscr{P}(\mathscr{E})$. For if $\mu(t)$ in (4.6) is a step function with jumps $\lambda_{j}$ at the points $t=t_{j}(j=1, \cdots, r)$ then by (4.8) the coefficients of $f(z)$ are

$$
a_{k}=\sum_{j=1}^{r} \lambda_{j} b_{k}\left(t_{j}\right), \quad k=1,2, \cdots
$$

Finally, we consider the coefficient space $\mathscr{A}_{n-1}$. By (4.8) and (4.4) we have that

$$
\operatorname{Re}\left\{a_{n}\right\}=\frac{2}{\cosh n c} \int_{0}^{2 \pi} \cos n t d \mu(t) .
$$

Lemma 4.1. If $\mu(t)$ is nondecreasing on $0 \leqq t \leqq 2 \pi, \quad \int_{0}^{2 \pi} d \mu(t)=1$, and $\mu(t+2 \pi)=\mu(t)+1$, then

$$
\int_{0}^{2 \pi}(1-\cos n t) d \mu(t) \geqq 0 .
$$

Equality in (4.12) holds only if $\mu(t)$ is constant in each of the intervals 


$$
k \frac{2 \pi}{n}<t<(k+1) \frac{2 \pi}{n}, \quad k=0,1, \cdots, n-1 .
$$

Proof. The inequality (4.12) is obvious. To prove the second assertion we assume for some $k, 0 \leqq k \leqq n-1$, that $\mu(t)$ is not constant on the interval $k(2 \pi / n)<t<(k+1)(2 \pi / n)$, then there exist numbers $\delta>0$ and $m=m(k, \delta)>0$ such that

$$
{ }^{\mu}\left[(k+1) \frac{2 \pi}{n}-\delta\right]>\mu\left[k \frac{2 \pi}{n}+\delta\right]
$$

and $1-\cos n t \geqq m$ when $k(2 \pi / n)+\delta \leqq t \leqq(k+1)(2 \pi / n)-\delta$. Thus

$$
\int_{0}^{2 \pi}(1-\cos n t) d \mu(t) \geqq m\left\{\mu\left[(k+1) \frac{2 \pi}{n}-\delta\right]-\mu\left[k \frac{2 \pi}{n}+\delta\right]\right\}>0,
$$

and inequality holds in (4.12).

Applying the lemma to (4.11) we have that

$$
\operatorname{Re}\left\{a_{n}\right\} \leqq \frac{2}{\cosh n c},
$$

and by (4.6), equality holds if, and only if,

$$
f(z)=\sum_{j=0}^{n-1} \lambda_{j} K\left(z, t_{j}\right), \quad t_{j}=j \frac{2 \pi}{n},
$$

where $\lambda_{j} \geqq 0, \sum \lambda_{j}=1$. Inequality (4.13) also follows from (4.2), however, the present proof shows that only the functions (4.14) are extremal for (4.13). Thus by (4.14), (4.3), and (4.4) the points in $\mathscr{A}_{n-1}$ are of the form

$$
\sum_{j=0}^{n-1} \lambda_{j}\left(b_{1}\left(t_{j}\right), \cdots, b_{n}\left(t_{j}\right)\right), \quad t_{j}=j \frac{2 \pi}{n},
$$

where $\lambda_{j} \geqq 0, \sum \lambda_{j}=1$, and

$$
b_{k}\left(t_{j}\right)=2 \cos \left(k t_{j}\right) / \cosh k c-i 2 \sin \left(k t_{j}\right) / \sinh k c .
$$

Note that $\sum_{j=0}^{n-1} \lambda_{j} b_{n}\left(t_{j}\right)=2 / \cosh n c$.

If we let $a_{v}=x_{v}+i y_{v}$, where

$$
a_{\nu}=\sum_{j=0}^{n-1} \lambda_{j} b_{\nu}\left(t_{j}\right)=\frac{2}{\cosh \nu c} \sum_{j=0}^{n-1} \lambda_{j} \cos \nu t_{j}-i \frac{2}{\sinh \nu c} \sum_{j=0}^{n-1} \lambda_{j} \sin \nu t_{j},
$$

then the points (4.15) are of the form $\left(x_{1}, y_{1}, x_{2}, y_{2}, \cdots, x_{n}, y_{n}\right)$. The variables $x_{v}, y_{v}$ satisfy the relations

$$
\begin{aligned}
& x_{\nu} \cosh \nu c=x_{n-\nu} \cosh (n-\nu) c, \\
& y_{\nu} \sinh \nu c=-y_{n-\nu} \sinh (n-\nu) c, \quad 1 \leqq \nu<n, \nu \leqq n-\nu,
\end{aligned}
$$


since

$$
x_{\nu} \frac{\cosh \nu c}{2}-i y_{\nu} \frac{\sinh \nu c}{2}=\sum_{j=0}^{n-1} \lambda_{j} e^{\left(i \nu_{j} 2 \pi / n\right)} .
$$

Thus $\mathscr{A}_{n-1}$ depends upon $n-1$ real variables $x_{v}, y_{\nu}$.

$$
\mathscr{A}_{n-1}=\mathscr{A}_{n-1}\left(x_{1}, y_{1}, x_{2}, y_{2}, \cdots, x_{(n-1) / 2}, y_{(n-1) / 2}\right)
$$

for $n$ odd $(n>1)$, and

$$
\mathscr{A}_{n-1}=\mathscr{A}_{n-1}\left(x_{1}, y_{1}, x_{2}, y_{2}, \cdots, x_{(n-2) / 2}, y_{(n-2 / 2}, x_{n / 2}\right)
$$

for $n$ even $(n>1)$. More precisely, $\mathscr{A}_{n-1}$ is a closed $(n-1)$-simplex with the $n$ vertices:

$$
\left(2 \cos \frac{j 2 \pi}{n} / \cosh c,-2 \sin \frac{j 2 \pi}{n} / \sinh c, 2 \cos \frac{j 4 \pi}{n} / \cosh 2 c,\right.
$$

$$
\begin{array}{r}
-2 \sin \frac{j 4 \pi}{n} / \sinh 2 c, \cdots, 2 \cos \frac{j(n-1) \pi}{n} / \cosh \frac{(n-1)}{2} c, \\
\left.-2 \sin \frac{j(n-1) \pi}{n} / \sinh \frac{(n-1)}{2} c\right),
\end{array}
$$

$j=0,1, \cdots, n-1, n$ odd $(n>1)$, and

$$
\left(2 \cos \frac{j 2 \pi}{n} / \cosh c,-2 \sin \frac{j 2 \pi}{n} / \sinh c, \cdots, 2 \cos \frac{(n-2) j \pi}{n} / \cosh \frac{(n-2)}{2} c,\right.
$$

$$
\left.-2 \sin \frac{(n-2) j \pi}{n} / \sinh \frac{(n-2)}{2} c, 2 \cos j \pi / \cosh \frac{n}{2} c\right)
$$

if $n$ is even.

The boundary hyperplanes of $\mathscr{A}_{n-1}$ have the equations $(k=0,1, \cdots, n-1)$

$$
1+\sum_{\nu=1}^{(n-1) / 2}\left(x_{\nu} \cosh \nu c \cos \frac{\nu k 2 \pi}{n}+y_{\nu} \sinh \nu c \sin \frac{\nu k 2 \pi}{n}\right)=0
$$

$n$ odd $(n>1)$, and

$$
\begin{aligned}
1+\frac{1}{2}( & -1)^{k} x_{n / 2} \cosh \frac{n c}{2} \\
& +\sum_{n=1}^{(n-2) / 2}\left(x_{\nu} \cosh \nu c \cos \frac{\nu k 2 \pi}{n}+y_{\nu} \sinh \nu c \sin \frac{\nu k 2 \pi}{n}\right)=0
\end{aligned}
$$

for $n$ even.

The hyperplanes (4.19) and (4.20) are tangent to the ellipsoids 


$$
\begin{array}{r}
\sum_{\nu=1}^{(n-1) / 2}\left(x_{\nu}^{2} \cosh ^{2} \nu c+y_{\nu}^{2} \sinh ^{2} \nu c\right)=\frac{2}{n-1}, \\
\frac{1}{2} x_{n / 2}^{2} \cosh ^{2} \frac{n c}{2}+\sum_{\nu=1}^{(n-2) / 2}\left(x_{\nu}^{2} \cosh ^{2} \nu c+y_{\nu}^{2} \sinh ^{2} \nu c\right)=\frac{2}{n-1},
\end{array}
$$

respectively.

We have proved the following:

THEOREM 4.3. Let the function

$$
f(z)=1+\sum_{n=1}^{\infty} a_{n} T_{n}(z)
$$

be regular and have positive real part in $\mathscr{E}$. Then $\operatorname{Re}\left\{a_{n}\right\} \leqq 2(\cosh n c)^{-1}$ and equality holds if, and only if, $f(z)$ is of the form (4.14). The coefficient space $\mathscr{X}_{n-1}$ corresponding to the extremal functions $(4.14)$ is a closed $(n-1)$-simplex consisting of points whose coordinates $x_{v}, y_{v}$ are determined by the equation (4.16). The vertices of the simplex are given by (4.17) and (4.18) and the boundary hyperplanes by the equations (4.19) and (4.20). The boundary hyperplanes are tangent to the ellipsoids (4.21).

\section{REFERENCES}

1. Ja. Ašnevic and G. Ulina, On value regions of analytic functions represented by a Stieltjes integral, Amer. Math. Soc. Transl. (2) 22 (1962), 81-94.

2. C. Carathéodory, Über den Variabilitätsbereich der Fourierschen Konstanten von positiven harmonischen Funktionen, Rend. Circ. Mat. Palermo 32 (1911), 193-217.

3. H. G. Eggleston, Convexity, Cambridge Univ. Press, Cambridge, 1958.

4. G. Goluzin, On a variational method in the theory of analytic functions, Amer. Math. Soc. Transl. (2) 18 (1961), 1-14.

5. G. Herglotz, Uber Potenzreihen mit positivem, reelem Teil im Einheitskreis, Ber. Verh. Sächs. Akad. Wiss. Leipzig Math.-Phys. Kl. 63 (1911), 501-511.

6. M. Robertson, Variational methods for functions with positive real part, Trans. Amer. Math. Soc. 102 (1962), 82-93.

7. _ Extremal problems for analytic functions with positive real part and applications, Trans. Amer. Math. Soc. 106 (1963), 236-253.

8. W. Royster, Functions having positive real part in an ellipse, Proc. Amer. Math. Soc. 10 (1959), 266-269.

9. , A Poisson integral formula for the ellipse and some applications, Proc. Amer. Math. Soc. 15 (1964), 661-670.

10. G. Szegö, Orthogonal polynomials, Amer. Math. Soc. Colloq. Publ. Vol. 23, p. 238, Amer. Math. Soc., Providence, R. I., 1939.

\footnotetext{
UNIVERSITY OF KENTUCKY

LEXINGTON, KENTUCKY

The University OF Kansas,

LAWRENCE, KaNSAS
} 